\title{
Reliability Sensitivity Analysis of Rub Impact in Coupled Fault Rotor Su Changqing ${ }^{1 \mathrm{a}}$, Guo fanyi ${ }^{2 \mathrm{~b}}$ and Shi qikun, ${ }^{2 \mathrm{c}}$ \\ ${ }^{1}$ School of Safety Engineering, Shenyang Aerospace University, Shenyang, China \\ ${ }^{2}$ School of Mechatronics Engineering, Shenyang Aerospace University, Shenyang, China \\ aneusucq@163.com, bsauguofy@163.com, csaushiqk@163.com
}

Keywords: pedestal looseness; rub impact; crack; reliability sensitivity; coupled fault rotor system Abstract. On the basis of the dynamic equations of rotor system with pedestal looseness, rub impact and crack, the random response method of rotor system with pedestal looseness is researched. The method is based on stochastic perturbation theory and the Kronecker algebra, matrix calculus and random perturbation are used. the reliability of rubbing for the coupling fault of rotor system was studied by Applying stress-strength interference theory and fourth-order moment technology, and on the basis of reliability analysis, the reliability sensitivity of rub impact on coupled fault rotor system is discussed by combining the sensitivity technology .

\section{Introduction}

Multiple fault often occur in the working process of rotating machinery at the same time, coupling faults can reduce the stiffness and strength of the rotor system, which will cause the occurrence of major accidents. So, research on the reliability sensitivity analysis of fault rotor system will have the theoretical and engineering-oriented significance for the rotating machinery[1].

In the paper, the model of rotor system with coupled faults has been built. On the basis of the rotor system model, the random response method of rotor system with coupled faults is presented.The first four moments of the response and the state function are obtained using the statistical fourth-moment method. The reliability sensitivity analysis method of rotor system with coupled faults is presented. Numerical results are also presented and discussed.

\section{Coupled fault rotor system model}

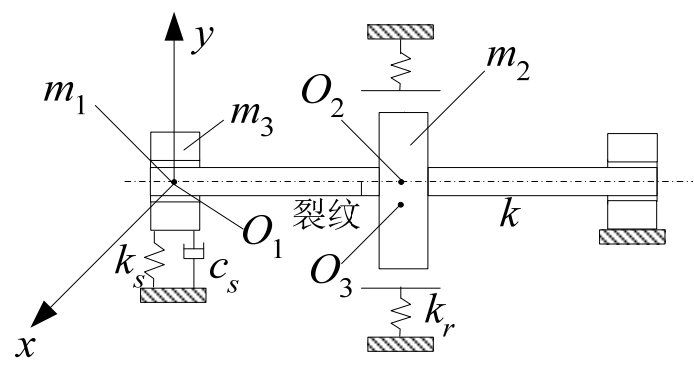

Fig.1 Rotor system

Consider a one-disk rotor system with coupled fault supported by two oil film bearing, as shown in Fig.1. $O_{1}$ is the geometric center of axle bush. $O_{2}$ is the geometric center of rotor. $O_{3}$ is the centroid of rotor. $e$ is the center imbalance distance of the rotor. $\mathrm{k}$ is the stiffness of elastic axes. $c_{1}$ is the damping coefficient of elastic axes. $c_{2}$ is the damping coefficient of disk. The lumped mass of rotor on bearing is $\mathrm{m} 1$. The lumped mass of rotor on disk is $\mathrm{m}_{2}$. The equivalent lumped mass of bearing is $\mathrm{m}_{3} . \omega$ is the rotor speed.

The axis displacement of non pedestal are denoted by $x_{1}, y_{1}$. The radial displacements of disk are denoted by $x_{2}, y_{2}$. The axis displacement of pedestal are denoted by $x_{3}, y_{3}$. The bearing base displacement in the vertical direction of pedestal is $\mathrm{y}_{4}$, so the governing equations can be written as 


$$
\left\{\begin{array}{l}
m_{1} \ddot{x}_{1}+c_{1} \dot{x}_{1}+k A\left(x_{1}-x_{2}\right)-B\left[\left(x_{1}-x_{2}\right) \cos (2 \omega t)+\left(y_{1}-y_{2}\right) \sin (2 \omega t)\right]=F_{x 1} \\
m_{1} \ddot{y}_{1}+c_{1} \dot{y}_{1}+k A\left(y_{1}-y_{2}\right)-B\left[\left(x_{1}-x_{2}\right) \sin (2 \omega t)-\left(y_{1}-y_{2}\right) \cos (2 \omega t)\right]=F_{y 1}-m_{1} g \\
m_{2} \ddot{x}_{2}+c_{2} \dot{x}_{2}+k A\left(2 x_{2}-x_{1}-x_{3}\right)-B\left[\left(2 x_{2}-x_{1}-x_{3}\right) \cos (2 \omega t)+\left(2 y_{2}-y_{1}-y_{3}\right) \sin (2 \omega t)\right]=P_{x}+m_{2} \omega^{2} e \cos \omega t \\
m_{2} \ddot{y}_{2}+c_{2} \dot{y}_{2}+k A\left(2 y_{2}-y_{1}-y_{3}\right)-B\left[\left(2 x_{2}-x_{1}-x_{3}\right) \sin (2 \omega t)-\left(2 y_{2}-y_{1}-y_{3}\right) \cos (2 \omega t)\right]=P_{y}+m_{2} \omega^{2} e \sin \omega t-m_{2} g \\
m_{1} \ddot{x}_{3}+c_{1} \dot{x}_{3}+k A\left(x_{3}-x_{2}\right)-B\left[\left(x_{3}-x_{2}\right) \cos (2 \omega t)+\left(y_{3}-y_{2}\right) \sin (2 \omega t)\right]=F_{x 3} \\
m_{1} \ddot{y}_{3}+c_{1} \dot{y}_{3}+k A\left(y_{3}-y_{2}\right)-B\left[\left(x_{3}-x_{2}\right) \sin (2 \omega t)-\left(y_{3}-y_{2}\right) \cos (2 \omega t)\right]=F_{y 3}-m_{1} g \\
m_{3} \ddot{y}_{4}+c_{s} \dot{y}_{4}+k_{s} y_{4}=-F_{y 4}-m_{3} g
\end{array}\right.
$$

where $A=(1-\varepsilon \alpha F(\psi)) / 2, B=k \varepsilon F(\psi) / 2, \varepsilon$ and $\alpha$ are the stiffness parameters related to the depth of crack. where $F x, F y$, is respectively nonlinear oil-film forces of sliding bearing in the $\mathrm{x}$ and $\mathrm{y}$ direction [2][3].

\section{Reliability analysis}

The limit-state Equation of rub impact is expressed as

$$
g(\delta, r)=\delta-r
$$

where the response, $r$, and the threshold, $\delta$ are independent random variables. The first fourth order moments of the state function $g(\delta, r)$ are determined as

$$
\begin{aligned}
& \mu_{g}=\mathrm{E}[g(\delta, r)]=\mathrm{E}(\delta)-\mathrm{E}(r)=\mu_{\delta}-\mu_{r} \\
& \sigma_{g}^{2}=\operatorname{Var}[g(\delta, r)]=\sigma_{\delta}^{2}+\sigma_{r}^{2} \\
& \theta_{g}=\mathrm{E}[g(\delta, r)-\bar{g}(\delta, r)]^{3}=\theta_{\delta}-\theta_{r} \\
& \eta_{g}=\mathrm{E}[g(\delta, r)-\bar{g}(\delta, r)]^{4}=\eta_{\delta}+\eta_{r}+6 \sigma_{\delta}^{2} \sigma_{r}^{2}
\end{aligned}
$$

The reliability index is defined as:

$$
\beta=\frac{3\left(\alpha_{4 g}-1\right) u_{g} / \sigma_{g}+\alpha_{3 g}\left(\mu_{g}^{2} / \sigma_{g}^{2}-1\right)}{\sqrt{\left(9 \alpha_{4 g}-5 \alpha_{3 g}^{2}-9\right)\left(\alpha_{4 g}-1\right)}}
$$

where $\alpha_{3 g}=\theta_{g} / \sigma_{g}^{3}, \alpha_{4 g}=\eta_{g} / \sigma_{g}^{4}$ respectively denote the coefficients of skewness and of kurtosis[4].

The transient reliability of the system is represented as

$$
R(\beta)=1-\Phi(-\beta)=\Phi(\beta)
$$

where $\Phi(\cdot)$ is the standard normal distribution function.

\section{Reliability sensitivity analysis}

The reliability sensitivity with respect to the mean value of the system random parameter is approximately derived as follows:

$$
\frac{\mathrm{D} R(\beta)}{\mathrm{D} \overline{\boldsymbol{B}}}=\frac{\partial R(\beta)}{\partial \beta} \frac{\partial \beta}{\partial u_{g}} \frac{\partial u_{g}}{\partial u_{r}} \frac{\partial u_{r}}{\partial \overline{\boldsymbol{B}}}
$$

Where

$$
\begin{gathered}
\frac{\partial R(\beta)}{\partial \beta}=\varphi(\beta) \quad \frac{\partial \beta}{\partial u_{g}}=\frac{3\left(\alpha_{4 g}-1\right) / \sigma_{g}+2 \alpha_{3 g} \mu_{g} / \sigma_{g}^{2}}{\sqrt{\left(9 \alpha_{4 g}-5 \alpha_{3 g}^{2}-9\right)\left(\alpha_{4 g}-1\right)}} \frac{\partial u_{g}}{\partial u_{r}}=-1 \\
\frac{\partial u_{r}}{\partial \overline{\boldsymbol{B}}}=\frac{u_{x} \frac{\partial u_{x}}{\partial \overline{\boldsymbol{B}}}+u_{y} \frac{\partial u_{y}}{\partial \overline{\boldsymbol{B}}}}{u_{r}} \frac{\partial u_{x}}{\partial \overline{\boldsymbol{B}}^{\mathrm{T}}}=\left[\begin{array}{llll}
\frac{\partial u_{x}}{\partial b_{1}} & \frac{\partial u_{x}}{\partial b_{2}} & \cdots & \frac{\partial u_{x}}{\partial b_{n}}
\end{array}\right] \frac{\partial u_{y}}{\partial \overline{\boldsymbol{B}}^{\mathrm{T}}}=\left[\begin{array}{llll}
\frac{\partial u_{y}}{\partial b_{1}} & \frac{\partial u_{y}}{\partial b_{2}} & \cdots & \frac{\partial u_{y}}{\partial b_{n}}
\end{array}\right]
\end{gathered}
$$


The reliability sensitivity with respect to the variance of the system random parameter is approximately derived as follows:

$$
\frac{\mathrm{D} R(\beta)}{\mathrm{D} \operatorname{Var}(\mathbf{B})}=\frac{\partial R(\beta)}{\partial \beta} \frac{\partial \beta}{\partial \sigma_{g}} \frac{\partial \sigma_{g}}{\partial \sigma_{r}} \frac{\partial \sigma_{r}}{\partial \operatorname{Var}(\mathbf{B})}
$$

Where

$$
\begin{aligned}
& \frac{\partial \sigma_{g}}{\partial \sigma_{r}}=\frac{\sigma_{r}}{\sigma_{g}} \quad \frac{\partial \sigma_{r}}{\partial \operatorname{Var}(\boldsymbol{B})}=\frac{\left(\frac{u_{x}}{u_{r}}\right)^{2} \sigma_{x} \frac{\partial \sigma_{x}}{\partial \operatorname{Var}(B)}+\left(\frac{u_{y}}{u_{r}}\right)^{2} \sigma_{y} \frac{\partial \sigma_{y}}{\partial \operatorname{Var}(B)}}{\sigma_{r}} \\
& \frac{\partial \sigma_{x}}{\partial \operatorname{Var}(\boldsymbol{B})}=\frac{1}{2 \sigma_{x}}\left[\frac{\partial u_{x}}{\partial \boldsymbol{B}} \otimes \frac{\partial u_{x}}{\partial \boldsymbol{B}}\right] \quad \frac{\partial \sigma_{y}}{\partial \operatorname{Var}(\boldsymbol{B})}=\frac{1}{2 \sigma_{y}}\left[\frac{\partial u_{y}}{\partial \boldsymbol{B}} \otimes \frac{\partial u_{y}}{\partial \boldsymbol{B}}\right]
\end{aligned}
$$

\section{Numerical example}

The random parameters of one rotor-bearing system $k, c_{1}, c_{2}, k_{\mathrm{r}}$, and $e$ are normally distributed with a coefficient of variation equal to 0.03 . The mean values of the parameters are $k=3.6 \times 10^{6}(\mathrm{~N} / \mathrm{m})$, $c_{1}=105(\mathrm{Ns} / \mathrm{m}), c_{2}=210(\mathrm{Ns} / \mathrm{m}), k \mathrm{r}=7.6 \times 10^{4}(\mathrm{~N} / \mathrm{m}), e=0.1(\mathrm{~mm})$, respectively. The first four order moments of the gap, $\delta$, are $3.0(\mathrm{~mm}), 0.451(\mathrm{~mm}), 0.028(\mathrm{~mm})^{3}, 0.0017(\mathrm{~mm})^{4}$. The deterministic parameters are $m_{1}=20(\mathrm{~kg}), m_{2}=80(\mathrm{~kg}), m_{3}=120(\mathrm{~kg}), f=0.12, \omega=2500(\mathrm{rad} / \mathrm{s}), \beta=\pi / 2, c_{\mathrm{s} 1}=80 \mathrm{~N} / \mathrm{mm}$, $c_{\mathrm{s} 2}=80 \mathrm{~N} / \mathrm{mm}, c_{\mathrm{s} 3}=120 \mathrm{~N} / \mathrm{mm}, k_{\mathrm{s} 1}=2.2 \times 10^{3} \mathrm{~N} / \mathrm{mm}, k_{\mathrm{s} 2}=1 \times 10^{3} \mathrm{~N} / \mathrm{mm}, k_{\mathrm{s} 3}=2.5 \times 10^{4} \mathrm{~N} / \mathrm{mm}$, respectively. The random parameter matrix is $\boldsymbol{B}=\left[k c_{1} c_{2} e k_{r}\right]$.

It is observed from from Fig. 3-12, reliability sensitivity to the mean of the random variables fluctuate in some moment, oscillation time point is basically the same, but fluctuated amplitude and trend really is not consistent. The reliability sensitivity for the mean value of $c_{2}, c_{1}, k$ and $k_{r}$ are positive, and the reliability sensitivity for the mean value of $e$ are negative, that is to say the reliability of rotor system increases as the mean value of $c_{2}, c_{1}, k$ and $k_{r}$, but descends as the mean value of $e$ rise. The reliability sensitivity for the variance of $c_{2}, c_{1}, k, k_{r}$ and $e$ are negative, that is to say the reliability of rotor system descends as the variance of $c_{2}, c_{1}, k, k_{r}$ and $e$ rise. This accords with the general knowledge of rotor-bearing system.

\section{Summary}

This paper presents a reliability analysis method for rub impact in coupled fault rotor system. The reliability model is defined. Techniques from random perturbation, frequency analysis and fourth moment method are employed to systematically resolve the reliability problem of coupled fault rotor system.

This paper also presents an approximate solution technique for the reliability sensitivity of rub impact in the coupled fault rotor system based on the fourth moment method. The influence of random parameters for the reliability sensitivity of coupled fault rotor system is studied. Numerical results are presented. 

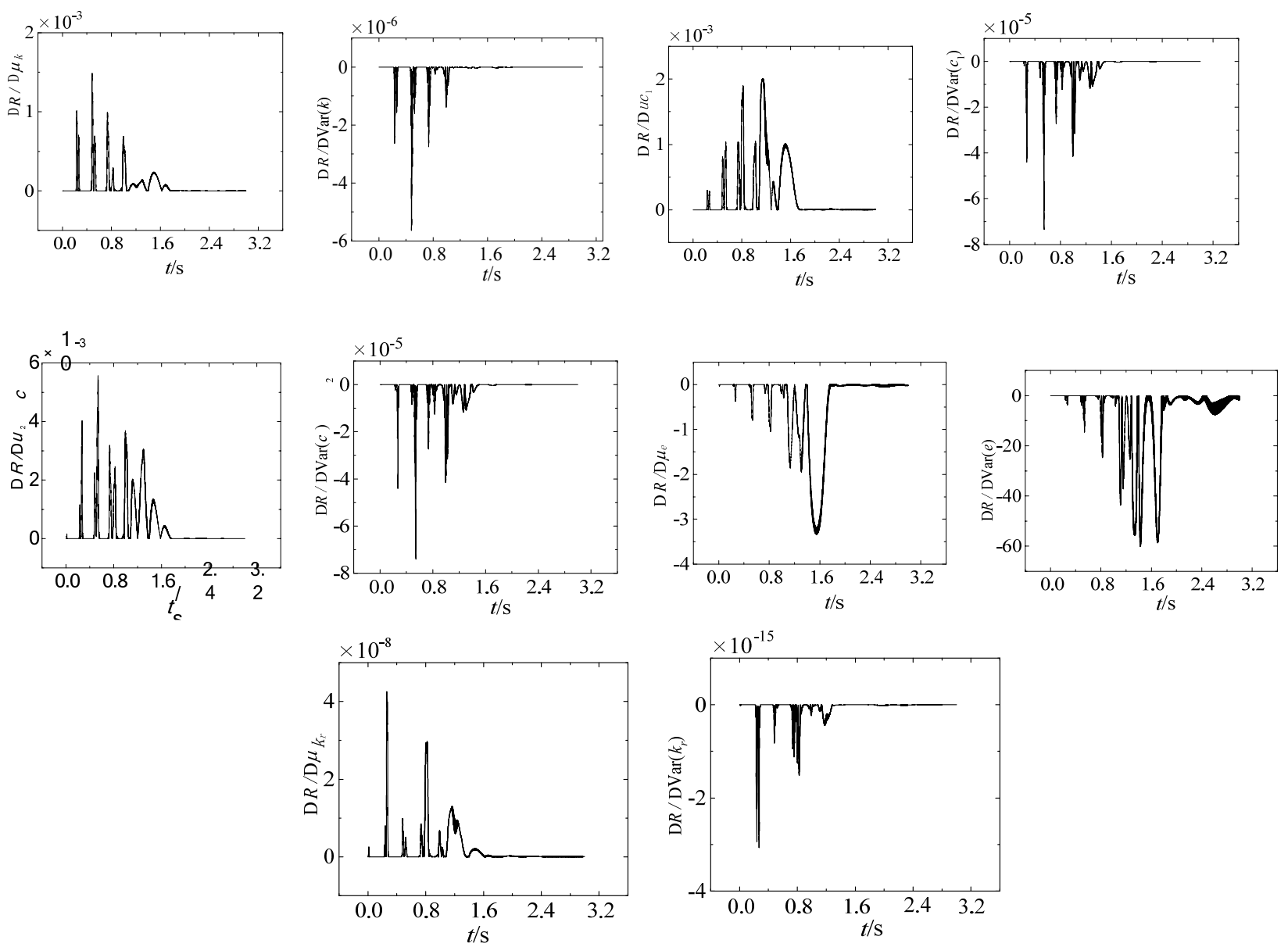

Fig.2 Reliability sensitivity curve

\section{Reference}

[1] Zhang Yi-min, Wen Bang-chun and Y. T Andrew. Leung: Journal of Vibration and Acoustics-Transactions of the ASME Vol.1(2002), p.58.

[2] Adiletta G, Guido A R and Rossi C:Nonlinear dynamics Vol.10(1996), p.251.

[3] YUAN Hui-qun, WEN Bang-chun and LI Hong-guang:Journal of Northeastern University Vol.6(2000), p.610.

[4] Zhao Y G and Ono T: Struct. Saf. Vol.6(2001), p.47. 